Naval Research Laboratory

Stennis Space Center, MS 39529-5004

NRL/FR/7440-03-10,053

\title{
Wave Bottom Boundary Layer Models for Smooth and Rough Beds
}

Jack Puleo

Mapping, Charting, and Geodesy Branch

Marine Geosciences Division

Oleg Mouraenko

University of Florida

Gainesville, Florida 32611-6550

September 17, 2003

Approved for public release; distribution is unlimited. 


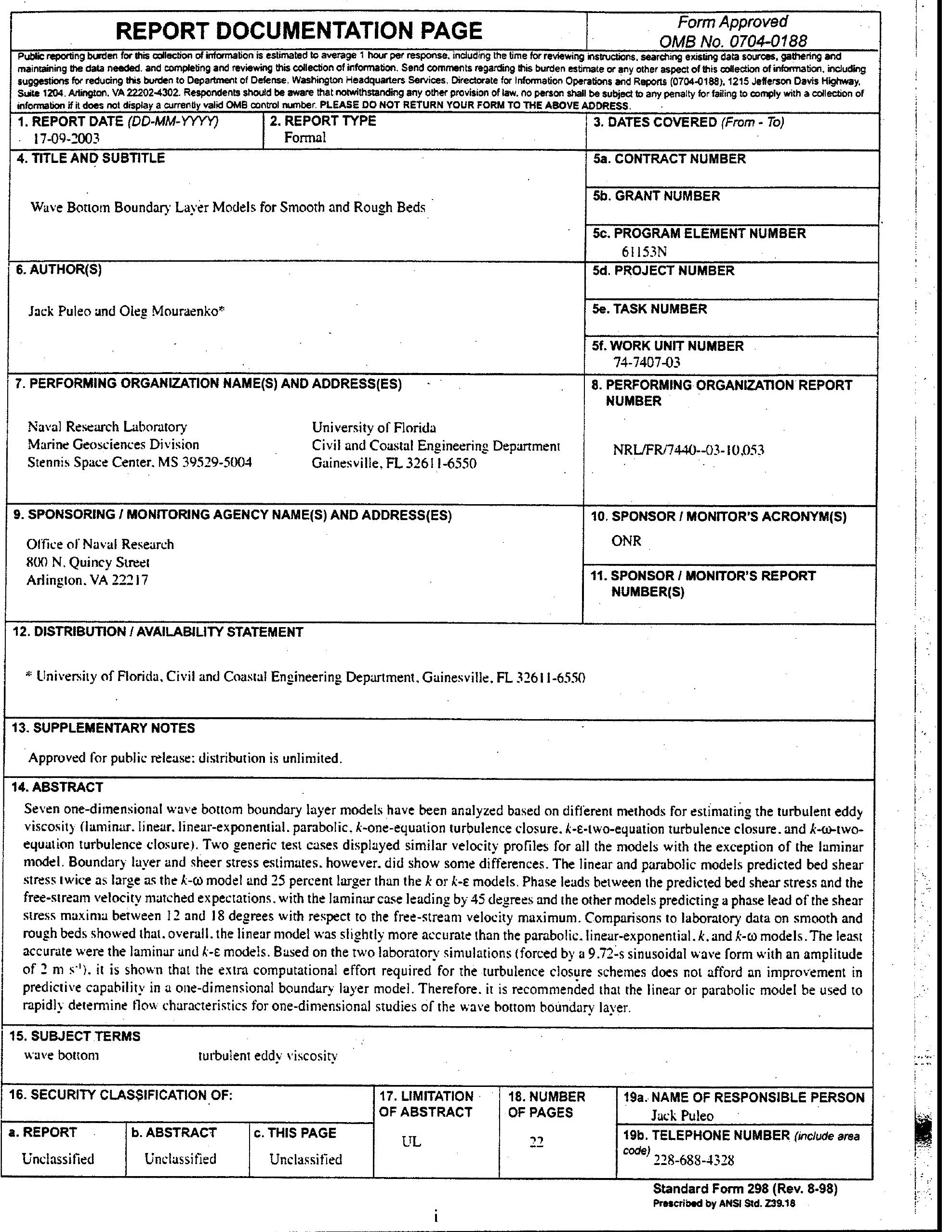




\section{CONTENTS}

INTRODUCTION

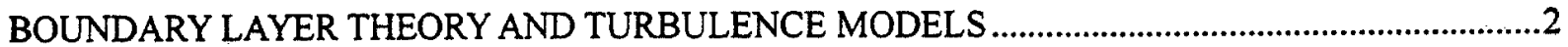

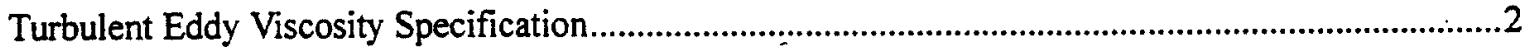

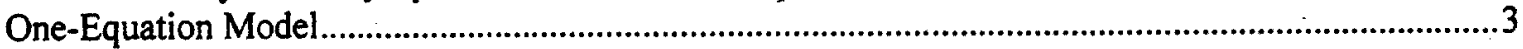

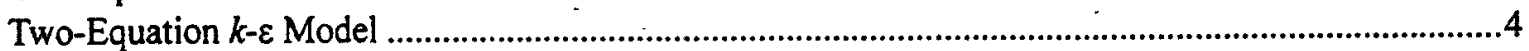

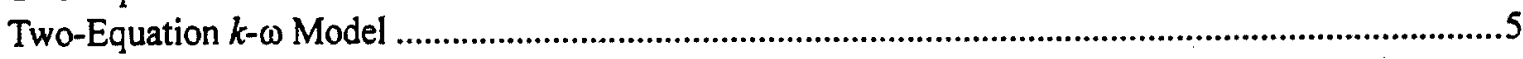

NUMERICAL METHODS

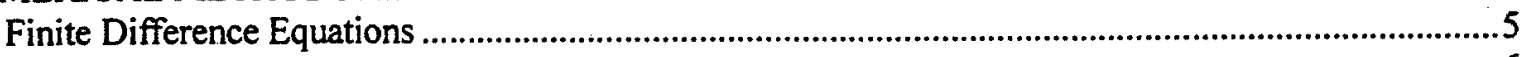

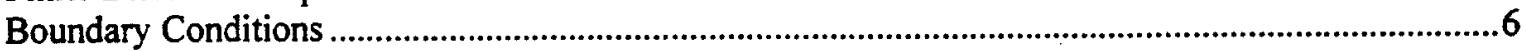

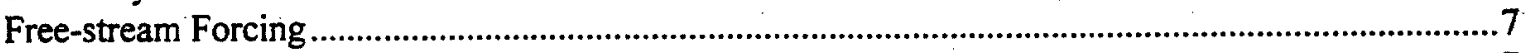

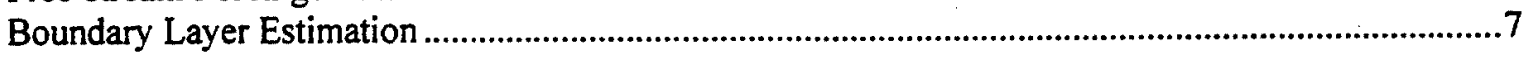

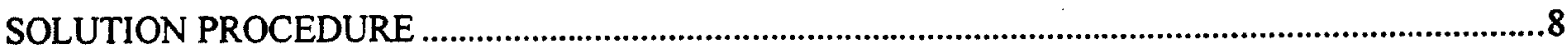

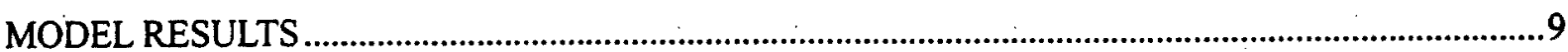

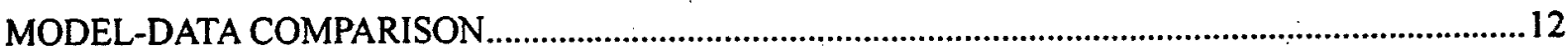

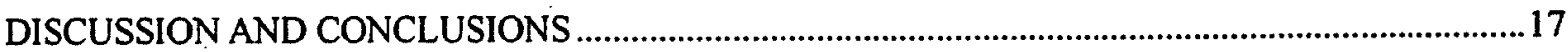

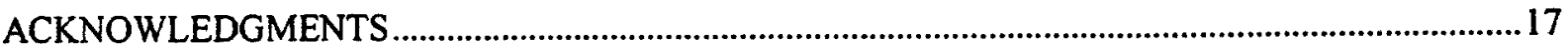

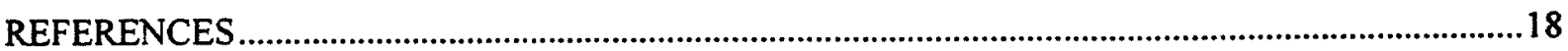

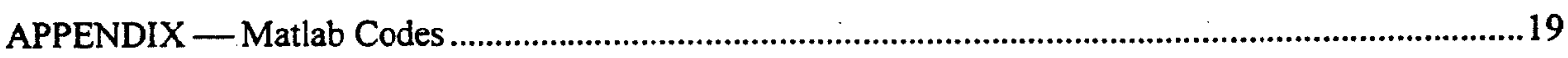




\section{WAVE BOTTOM BOUNDARY LAYER MODELS FOR SMOOTH AND ROUGH BEDS}

\section{INTRODUCTION}

The goal of the 6.1 Foreshore Sediment Transport Study is to develop predictive capabilities for the hydrodynamics and sediment transport in the inner surf and swash zones using field data and numerical models. The sediment transport aspect of the study often stems from some prediction of the fluid-supplied bed-shear stress resulting from friction and turbulent fluctuations near the bed in what is termed the wave bottom boundary layer (WBBL). Hence, knowledge of the time-dependent nature of fluid flows in this region will lead to better predictions of the turbulent motions and shear stresses and ultimately assist in improving sediment transport formulations in wave-dominated environments.

Much of the work on WBBLs has focused on developing various forms for the vertical distribution of the turbulent eddy viscosity (generically referred to here as eddy viscosity models), an approximation assuming that turbulent motions diffuse momentum in a manner similar to that of a molecular diffusive process although generally at a much faster rate. The form of the vertical distribution has varied from linear to parabolic to combined forms that do not have a single analytic function describing them. For instance, Fredsøe and Deigaard (1992) discuss several forms for the eddy viscosity, including those that can be adapted to independently account for a superimposed current.

A more detailed approach can be used by trying to calculate, rather than model, the turbulent motions themselves. For instance, Spalart (1988) and Moneris and Slinn (submitted) used a direct numerical simulation (DNS), such that all scales of turbulent motion were calculated, rendering a description of the turbulent eddy viscosity unnecessary. While DNS simulations are probably the most accurate for determining the fluid motions in the WBBL, their extensive computation requirements make them unusable for rapid estimations of the turbulent fluid motions in this region.

Rather than specifying the shape of the turbulent eddy viscosity or calculating the fluid motions at all scales, a middle ground approach can be taken where the turbulent motions are modeled leading to a time and space varying eddy viscosity (generically referred to here as turbulence closure models). One approach is referred to as the one-equation or $k$ (turbulent kinetic energy) model. Here the turbulent kinetic energy fluctuations are modeled and the eddy viscosity is estimated by specifying some length scale of the turbulence based on the elevation above the bed (e.g., Wilcox 2000). A second approach is to use a two-equation model where the length scale is no longer held constant and itself is allowed to vary (e.g., Rodi 1980, Justesen 1988, and Wilcox 1988).

The purpose of this report is to analyze seven WBBL models incorporating various methods for estimating the turbulent eddy viscosity that can be used to rapidly predict the fluid flow structure and shear stress occurring near the bed. The models will be compared using two test cases and against a

Manuscript approved April 18, 2003. 
laboratory data set. The report includes an appendix with the computer codes used (on disk), and a short description of how to run the simulations.

\section{BOUNDARY LAYER THEORY AND TURBULENCE MODELS}

The time-dependent equation of turbulent motion for an incompressible Newtonian fluid with an ensemble mean oscillatory velocity component $u$ parallel to the bed is given by

$$
\frac{\partial u}{\partial t}=-\frac{1}{\rho} \frac{\partial p}{\partial x}+\frac{\partial}{\partial z}\left[\left(v+v_{t}\right) \frac{\partial u}{\partial z}\right]
$$

where $\rho$ is the fluid density, $p$ is the pressure, $v$ is the kinematic viscosity of the fluid, $v$, is the turbulent eddy viscosity, $x$ is the horizontal coordinate, and $z$ is the vertical coordinate. .

Outside the boundary layer, in the free stream, shear stresses are no longer present such that there is a balance between the time-dependent free-stream velocity and the horizontal pressure gradient as

$$
\frac{\partial U}{\partial t}=-\frac{1}{\rho} \frac{\partial p}{\partial x}
$$

where $U$ is the free-stream velocity.

Substituting Eq. (2) into Eq. (1) and rearranging yields

$$
\frac{\partial u_{d}}{\partial t}=\frac{\partial}{\partial z}\left[\left(v+v_{t}\right) \frac{\partial u_{d}}{\partial z}\right],
$$

where the deflect velocity, $u_{d}=u-U$, represents the difference between the depth-dependent velocity and
that of the free stream.

A variety of methods have been arrived at to determine or specify $v_{i}$. One option is to assume or require the flow be laminar, in which case $v_{t}$ is zero for all time. Many other options exist, several of
which are outlined below.

\section{Turbulent Eddy Viscosity Specification}

Borrowing from open channel flow, one can specify the shape of the turbulent eddy viscosity a priori as some function depending on the height above the bed and the friction velocity at the bed. The height dependence arises because turbulent eddy size is restricted as the bed is approached. Friction velocity dependence arises to incorporate the shear at the bed (and hence a measure of the strength of the flow).

The linear form

$$
v_{t}=\kappa u_{*} z
$$


where $\kappa$ is the von Karman's constant $(=0.4)$ and $u_{*}$ is the friction velocity was first used by, for example, Jonsson (1966), Kajiura (1968), and Grant and Madsen (1979), allowing for an analytic solution to Eq. (3).

The friction velocity is given as

$$
u_{*}=\sqrt{\frac{\tau}{\rho}}
$$

and the shear stress $\tau$ is, in turn, defined as

$$
\tau=\rho\left(v+v_{1}\right) \frac{\partial u}{\partial z}
$$

Equation (6) is often seen without the kinematic viscosity term, however we include it since the flows will typically begin from rest and some form of shear near the bed will still exist on the molecular level.

The linear form gives the expected behavior of the turbulent eddy viscosity near the bed, but is physically incorrect near the surface. The turbulent eddies near the surface are predicted to be too large according to Eq. (4), since it is expected that turbulent shear stresses vanish outside the boundary layer (assuming no mean currents are superimposed).

To relieve some of this difficulty, Gelfenbaum and Smith (1986) modified the linear turbulent eddy viscosity by including an exponential term that decays with increasing elevation above the bed. Beach and Sternberg (1988) further modified the linear-exponential form to

$$
v_{t}=\kappa u_{*} z e^{-2 * z / D}
$$

where $D$ is the domain depth in their study of sediment suspension, assuming that the diffusion of mass and momentum were equal.

Finally, Fredsøe and Deigaard (1992) give a parabolic form of the turbulent eddy viscosity as

$$
v_{t}=\kappa u_{*} z\left(1-\frac{z}{D}\right)
$$

It may expected that this latter form would be more correct compared to Eqs. (7) and (4) since it has roughly the same shape near the bed but goes to zero at the surface. Equation (8) can also be modified such that $D$ is replaced with the boundary layer height.

\section{One-Equation Model}

Rather than specifying the form of the eddy viscosity, it can be estimated based on the turbulent kinetic energy in the flow field. The turbulent kinetic energy $k$ for a one-dimensional problem is defined as

$$
k=\frac{1}{2} u_{d}^{2}
$$


where $u_{d}^{\prime}$ are the turbulent fluctuations from the mean velocity. The transport equation for $k$ is derived in, for example, Fredsøe and Deigaard (1992), and given as

$$
\frac{\partial k}{\partial t}=\frac{\partial}{\partial z}\left[\left(v+v_{t}\right) \frac{\partial k}{\partial z}\right]+v_{t}\left(\frac{\partial u_{d}}{\partial z}\right)^{2}-C_{1} \frac{k^{3 / 2}}{L},
$$

where $C_{l}$ is a constant $(=0.09)$ and $L$ is a turbulence length scale given by

$$
L=\kappa \sqrt[4]{C_{1}} z
$$

In Eq. (10), the time rate of change of turbulent kinetic energy results from diffusion (both molecular and turbulent), production (note there is no molecular production of turbulent kinetic energy, hence $v$ is not included in this term), and dissipation. Finally, the eddy viscosity results from the KolmogorovPrandtl relationship between eddy viscosity, a mixing length and the turbulent kinetic energy as

$$
v_{t}=L \sqrt{k}
$$

\section{Two-Equation $k-\varepsilon$ Model}

Similar to the one-equation model, the turbulent kinetic energy is modeled through an equation similar to Eq. (10) in the two-equation model. The turbulence length scale, however, is allowed to vary in time and space rather than remain fixed as in the one-equation model. In order for $L$ to vary, an additional equation is required to model the dissipation of turbulence $\varepsilon$, leading to the so-called $k-\varepsilon$ equations as (see Justesen 1988 or Wilcox 2000 for the complete derivation):

$$
\frac{\partial k}{\partial t}=\frac{\partial}{\partial z}\left[\left(v+\frac{v_{t}}{\sigma_{k}}\right) \frac{\partial k}{\partial z}\right]+v_{t}\left(\frac{\partial u_{d}}{\partial z}\right)^{2}-C_{1} \varepsilon
$$

and

$$
\frac{\partial \varepsilon}{\partial t}=\frac{\partial}{\partial z}\left[\left(v+\frac{v_{t}}{\sigma_{\varepsilon}}\right) \frac{\partial \varepsilon}{\partial z}\right]+C_{2} v_{t} \frac{\varepsilon}{k}\left(\frac{\partial u_{d}}{\partial z}\right)^{2}-C_{1} C_{3} \frac{\varepsilon^{2}}{k}
$$

The use of $C_{1}$ in the dissipation terms follows Justesen (1988) and is a simplified method of reducing the dissipation rather than using empirical functions (see Hwang and Jaw (1998) for a description of these various functions).

The constants used in Eqs. (10), (13), and (14) are often found from direct numerical simulations with the typical values given in Table 1 .

Table 1 - Constants Used in the $k-\varepsilon$ Model

\begin{tabular}{|l|l|l|l|l|}
\hline$C_{l}$ & $C_{2}$ & $C_{3}$ & $\sigma_{k}$ & $\sigma_{\varepsilon}$ \\
\hline 0.09 & 1.44 & 1.92 & 1.0 & 1.3 \\
\hline
\end{tabular}


Upon determining $k$ and $\varepsilon$, the turbulent eddy viscosity is determined by

$$
v_{t}=C_{1} \frac{k^{2}}{\varepsilon}
$$

It is known that the $k-\varepsilon$ model has particular difficulty in complex flows and those with separation points and adverse pressure gradients (Rodi and Scheuerer 1986, Pope 2000, and Wilcox 2000). Hence, it should be used in direction-reversing boundary layer flows with caution.

\section{Two-Equation $k-\omega$ Model}

Like the $k-\varepsilon$ model, the $k-\omega$ model, which is known to be more robust than the $k-\varepsilon$ in regions of adverse pressure gradients (e.g., Wilcox 2000), is a two-equation turbulence closure scheme except that the second equation models the specific dissipation rate $\omega(=\varepsilon / k)$, rather than the dissipation rate $\varepsilon$.

The model equations (Wilcox 1988) are

$$
\begin{aligned}
& \frac{\partial k}{\partial t}=\frac{\partial}{\partial z}\left[\left(v+\sigma^{*} v_{t}\right) \frac{\partial k}{\partial z}\right]+v_{t}\left(\frac{\partial u_{d}}{\partial z}\right)^{2}-\beta^{*} k \omega, \\
& \frac{\partial \omega}{\partial t}=\frac{\partial}{\partial z}\left[\left(v+\sigma v_{t}\right) \frac{\partial \omega}{\partial z}\right]+\alpha v_{t} \frac{\omega}{k}\left(\frac{\partial u_{d}}{\partial z}\right)^{2}-\beta \omega^{2}
\end{aligned}
$$

and

$$
v_{t}=\frac{k}{\omega}
$$

and the coefficients are given in Table 2.

Table 2 - Constants Used in the $k$ - $\omega$ Model

\begin{tabular}{|l|l|l|l|l|}
\hline$\sigma$ & $\sigma^{*}$ & $B$ & $B^{*}$ & $\alpha$ \\
\hline 0.5 & 0.5 & $3 / 40$ & $9 / 100$ & $5 / 9$ \\
\hline
\end{tabular}

\section{NUMERICAL METHODS}

\section{Finite Difference Equations}

The equations for the boundary layer flow and the various eddy viscosity approaches are solved numerically via an implicit algorithm using $2^{\text {nd }}$ order spatial differences using a logarithmically spaced grid. The implicit Euler method is given as (using deflect velocity as an example)

$$
\frac{\partial u}{\partial t}=\frac{u_{d}^{n+1}-u_{d}^{n}}{\Delta t}
$$

where the superscript is the time level. Second order spatial differences are given as 


$$
\frac{\partial u_{d}}{\partial z}=\frac{h_{i}-h_{i-1}}{h_{i-1} h_{i}} u_{d, i}+\frac{1}{h_{i-1}+h_{i}}\left[\frac{h_{i-1}}{h_{i}} u_{d, i+1}-\frac{h_{i}}{h_{i-1}} u_{d, i-1}\right]
$$

and

$$
\frac{\partial^{2} u_{d}}{\partial z^{2}}=\frac{2}{h_{i-1}+h_{i}}\left[\frac{u_{d, i+1}}{h_{i}}+\frac{u_{d, i-1}}{h_{i-1}}\right]-\frac{2 u_{d, i}}{h_{i-1} h_{i}},
$$

where $i$ is the grid point location and

$$
h_{i}=z_{i+1}-z_{i}
$$

Systems of equations are developed upon substituting Eqs. (19) through (22) for the various variables into the model equations.

\section{Boundary Conditions}

In order to fully specify the problem, boundary conditions are needed for Eqs. (10), (13), (14), (16), and (17). For the velocity, the boundary condition imposed at the bed is no slip such that the deflect velocity at the roughness level (the first grid point) is

$$
u_{d}\left(\frac{k_{N}}{30}, t\right)=-U(t)
$$

where $k_{N}$ is the Nikuradse equivalent sand roughness and $z_{0}=k_{N} / 30$ is the roughness length where the fluid velocity goes to zero. The velocity gradient is assumed to vanish at the top of the domain, leaving

$$
\frac{\partial u_{d}}{\partial z}(D, t)=0
$$

The boundary conditions for $k$ are

$$
k\left(\frac{k_{N}}{30}, t\right)=\frac{1}{\sqrt{C_{1}}} v_{t}\left|\frac{\partial u_{d}}{\partial z}\right|
$$

and

$$
\frac{\partial k}{\partial z}(D, t)=0
$$

The lower boundary condition on $k$ stems from the fact that the turbulent velocity fluctuations need not go to zero on a hydraulically rough bed. Moreover, equilibrium is assumed to exist between the production of turbulent kinetic energy and dissipation, yielding Eq. (27); see Justesen (1988). Rodi (1980) gives the boundary conditions for the dissipation equation as

$$
\varepsilon\left(\frac{k_{N}}{30}, t\right)=C_{1}^{3 / 4} \frac{k^{3 / 2}}{k z_{0}}
$$

and 


$$
\frac{\partial \varepsilon}{\partial z}(D, t)=0
$$

The bottom boundary condition for the specific dissipation rate is (Wilcox 2000)

$$
\omega\left(\frac{k_{N}}{30}, t\right)=\frac{k^{1 / 2}}{\left(\dot{\beta}^{*}\right)^{1 / 4} \kappa z_{0}} .
$$

Solutions are sensitive to the value of a Dirichlet free stream boundary condition so we apply a Neumann condition at the surface similar to the conditions for $k$ and $\varepsilon$.

$$
\frac{\partial \omega}{\partial z}(D, t)=0
$$

\section{Free-Stream Forcing}

The models can be forced by either a time series of free stream velocity measurements or any analytic wave representation with or without a superimposed current. Analytic wave representations used in this study are sinusoidal and sawtooth waves. Sinusoidal forcing is given by

$$
U(t)=U_{m} \sin \left(\frac{2 \pi t}{T}\right),
$$

where $U_{m}$ is the maximum free stream flow amplitude and $T$ is the sinusoidal wave period. Sawtooth waves can be forced using the form described by Fredsøe and Deigaard (1992) as

$$
U(t)=\left\{\begin{array}{l}
U_{m} \frac{12 t}{\alpha} 0<t<\alpha_{s} \frac{T}{2} \\
U_{m} \frac{1-2 \frac{t}{T}}{1-\alpha_{s}} \alpha_{s} \frac{T}{2} \leq t<\frac{T}{2}
\end{array},\right.
$$

where $\alpha_{s}$ is a parameter controlling the skewness of the wave and $T$ is the sawtooth wave period.

\section{Boundary Layer Estimation}

The boundary layer height is calculated in one of two ways as

$$
\delta=z \mid \frac{u_{d}+U}{U}=P_{\delta} \text { (hereinafter referred to as } \dot{B L} 1 \text { ) }
$$

where $u_{d}+U$ is the depth-dependent velocity. $P_{\delta}$ is the ratio of the velocity to that of the free stream and is usually set to 0.95 or 0.99 ; the location where this occurs is defined as the boundary layer thickness $\delta$. Difficulties can arise with Eq. (33) because the free-stream velocity goes through zero when forced by 
oscillatory and aperiodic direction-reversing flows. Hence, a second option for calculating the boundary layer thickness is to determine when the magnitude of the gradient of $u_{d}$ passes a tolerance threshold as

$$
\delta=z|| \frac{\partial u_{d}}{\partial z} \mid \geq P_{\delta z} \text { (hereinafter referred to as BL2). }
$$

Starting at the surface and moving in the downward direction, the location where the magnitude of the velocity gradient meets or exceeds $P_{\delta z}$ specifies the height of the boundary layer. This method can produce realistic boundary layer heights even during times of zero or near-zero flow above the boundary layer.

\section{SOLUTION PROCEDURE}

The models (laminar, linear, linear-exponential, parabolic, $k, k-\varepsilon$, and $k-\omega$ ) have been coded in Matlab using the implicit numerical methods mentioned above. Unfortunately, the models represent coupled systems of equations relying on an unknown $(n+1)$ time step. The implicit solution procedure for the turbulence closure models requires iteration on the variables $\left(u_{d}, k, u_{d}, k, \varepsilon\right.$; or $\left.u_{d}, k, \omega\right)$ to obtain a converged solution at each time step. The eddy viscosity models do not require iteration.

Figure 1 shows a flow chart of the solution procedure. After defining the variables in the workspace and the initial conditions, the deflect velocity is solved for and the vertical derivative of the deflect velocity determined. In the case of the linear, linear-exponential or parabolic model, the friction velocity is next found and used to update the turbulent eddy viscosity for the next time step. For the one-equation model, the $k$ value at time $n+1$ is determined and used to update the turbulent eddy viscosity using the length scale through Eq. (12). These steps are repeated for this time step until the error on the turbulent eddy viscosity is smaller than an iteration tolerance. In the two-equation models, the $k$ equation is solved, followed by the $\varepsilon$ or $\omega$ equation, and, finally, the turbulent eddy viscosity is calculated through Eq. (15) or (18). Similar to the one-equation model, these steps are iterated upon until the error in the turbulent eddy viscosity is smaller than an iteration tolerance. The variables are then updated for the next time step, the boundary layer thickness and bed shear stress are calculated, and the next time step is initiated.

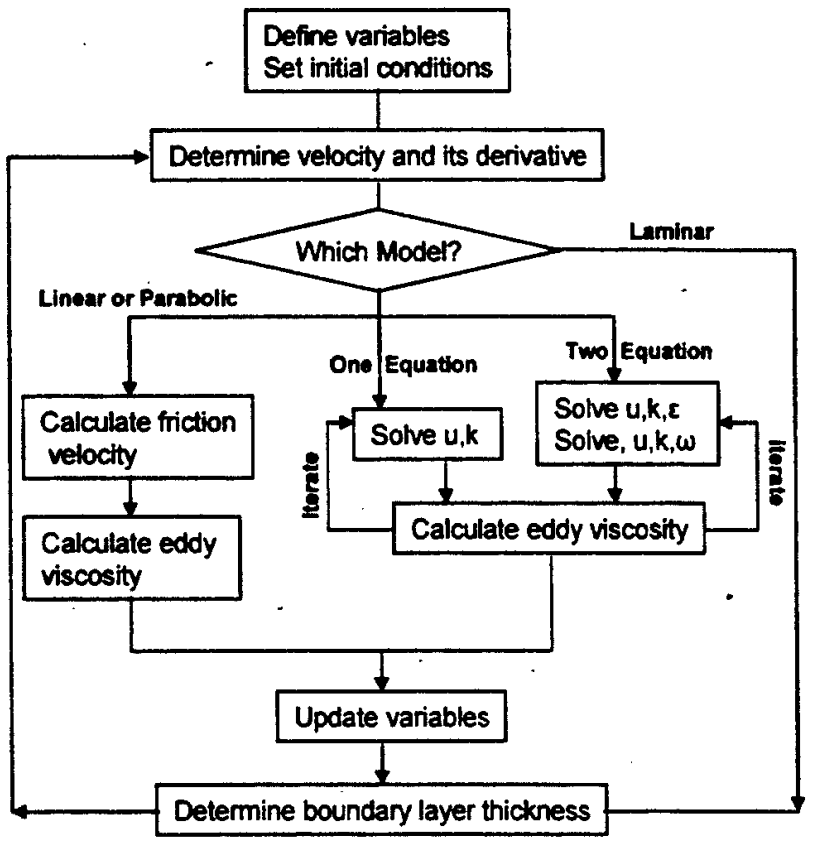

Fig. 1 -Flow diagram of boundary layer model solution procedure 


\section{MODEL RESULTS}

All seven models were tested against each other for two generic cases. (The linear-exponential model is not shown in any of the figures because of the similarity to the linear and parabolic eddy viscosity model.) The first case (CASEl) is a 5-s sine wave with an amplitude of $0.8 \mathrm{~m} \mathrm{~s}^{-1}$. The second case (CASE2) is a 5-s sawtooth wave with an amplitude of $0.8 \mathrm{~m} \mathrm{~s}^{-1}$ and $\alpha_{s}=0.25$. In both cases, $k_{N}=1 \times 10^{-4}$ $\mathrm{m}$, the domain height is $0.1 \mathrm{~m}$, the grid spacing was specified as logarithmic (with 300 points), and 2000 time steps were completed for each of four wave periods (only the last was retained for comparison).

Figure 2 shows velocity from four phases (at $0^{\circ}, 45^{\circ}, 90^{\circ}$, and $135^{\circ}$ ) of the CASEl comparison. At $0^{\circ}$, the flow has reversed from negative to positive, and the phase lead of the boundary layer flow can be seen in all cases. The laminar simulation predicts a very small boundary layer height and the largest near bed velocity at over $0.25 \mathrm{~m} \mathrm{~s}^{-1}$. The other simulations have similar (to each other) near-bed velocity maxima of about $0.18 \mathrm{~m} \mathrm{~s}^{-1}\left(0.22 \mathrm{~m} \mathrm{~s}^{-1}\right.$ for $k-\varepsilon$ model) but vary higher in the water column. The $k-\varepsilon$ and $k-\omega$ velocity predictions decrease with elevation more rapidly than the $k$, linear, or parabolic simulations (note that the linear and parabolic simulations are nearly identical). Also, these two simulations still predict negative flow from about $0.02 \mathrm{~m}$ to $0.045 \mathrm{~m}$, whereas the linear, parabolic, and $k$ simulations do not.
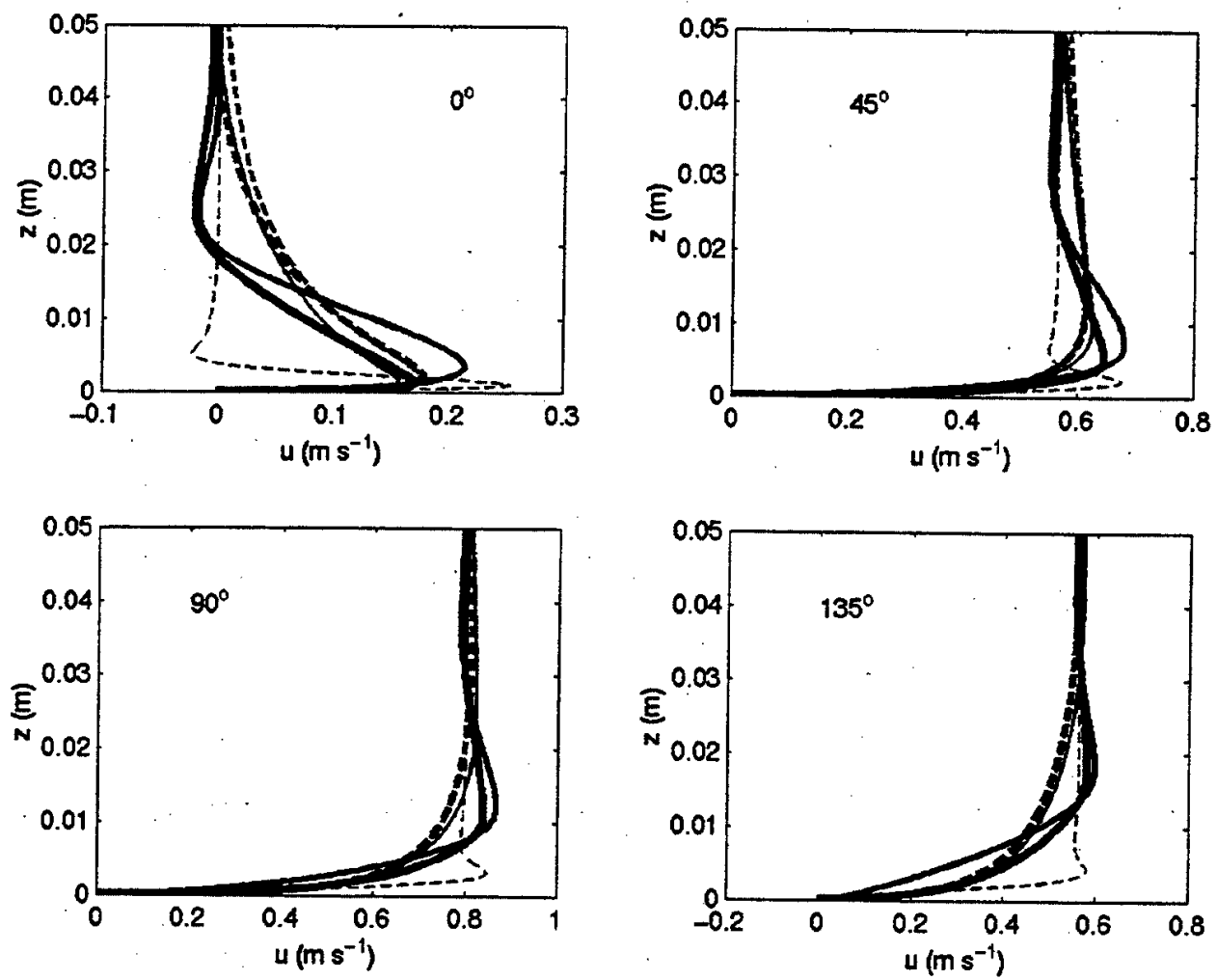

Fig. 2 - CASE1 velocity profiles for various wave phases: laminar (.............); linear (........); parabolic ( $=\square) ; k(\longrightarrow) ; k-\varepsilon(\square)$; and $k-\omega(\omega)$.

At $45^{\circ}$, when the flow is accelerating, all simulations except the laminar one have essentially the same structure with small variations. For instance, the $k$, linear, and parabolic models do not have an inflection point near $z=0.02 \mathrm{~m}$. At $90^{\circ}$, the acceleration is 0 and the free-stream velocity is at its maximum. Again, the simulations look similar with only subtle differences. For instance, the $k-\varepsilon$ and $k-\omega$ velocities increase with respect to elevation faster than do the other simulations (except laminar). Finally, by $135^{\circ}$, the flow 
is decelerating. Note the difference in vertical structure between this plot and that for $45^{\circ}$. Both have the same free-stream velocity, but the flow at $45^{\circ}$ is accelerating while the flow at $135^{\circ}$ is decelerating.

Figure 3 shows the CASE2 profiles. At $0^{\circ}$, the shapes of the vertical profiles do not vary much from their CASE1 counterparts. The laminar model, as before, predicts a much thinner boundary layer and larger near-bed velocity. At $45^{\circ}$ and $90^{\circ}$, the shapes of the profiles are similar to those of CASEl, but the velocity magnitude is reduced by roughly one half and one fourth, respectively, due to the skewed forcing of the sawtooth wave. The wave skewness, however, causes the velocity maxima at $135^{\circ}$ to exceed those in CASE1 by over $0.2 \mathrm{~m} \mathrm{~s}^{-1}$.
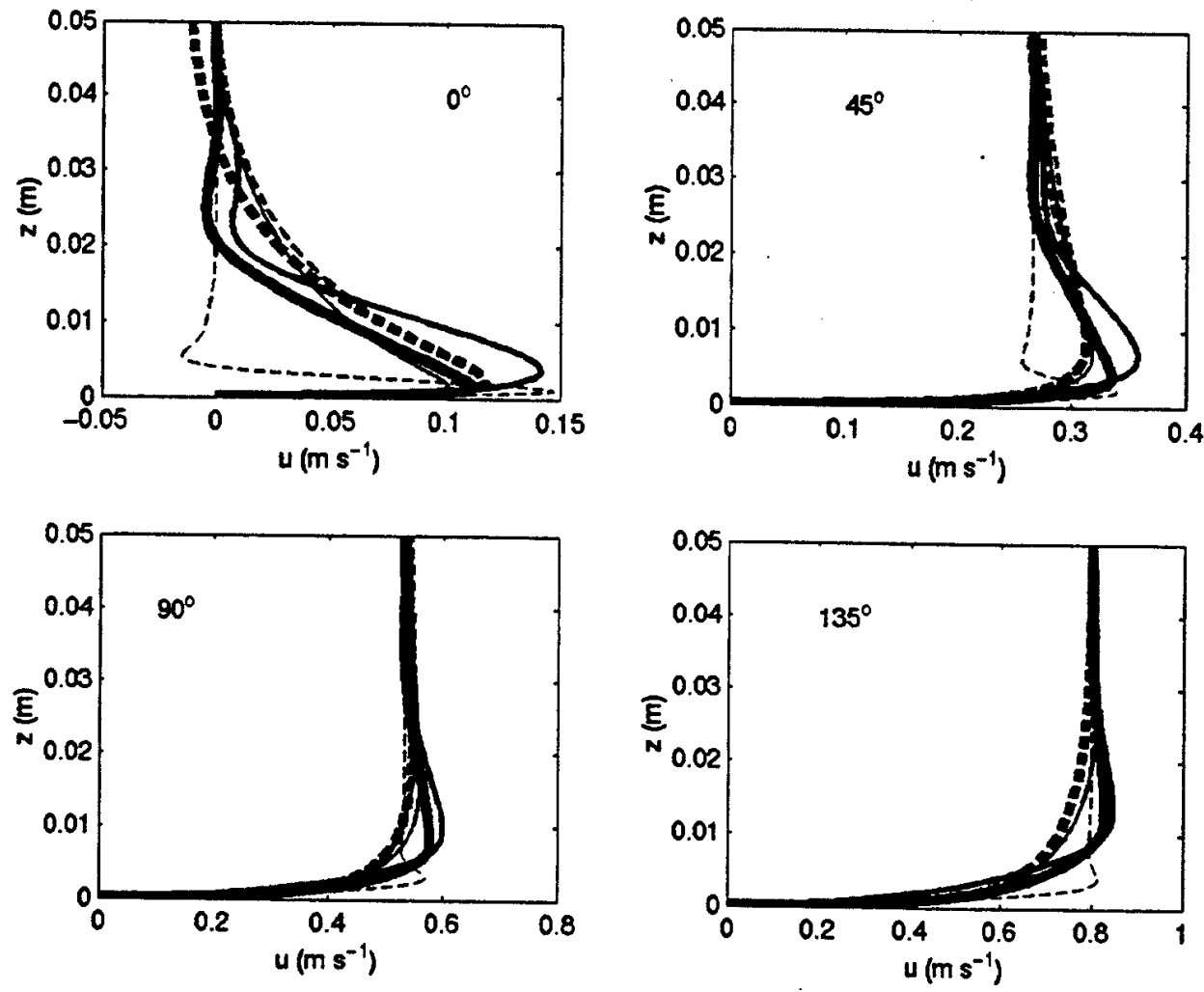

Fig. 3 - CASE2 velocity profiles for various wave phases: laminar (..............); linear (.......);

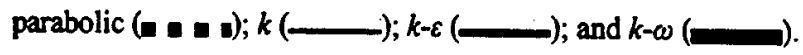

Figure 4 shows the boundary layer estimations based on the two methods (BL1 and BL2) for CASE1. The advantage of BL2 (Fig. 4A) over BL1 (Fig. 4B) is clearly seen at flow reversals when the free-stream velocity passes through zero, and the BLl estimate reaches the domain height. While the boundary layer estimation using the velocity gradient appears to perform better, there are still considerable differences in the shapes between the various simulations. Decreases in the estimated boundary layer height are seen for the $k-\varepsilon$ and $k-\omega$ simulations near-flow reversal, while decreases are seen for the other nonlaminar simulations closer to peak free-stream velocity magnitudes. Other estimations for the BL2 method can be obtained by varying the cutoff $P_{\delta=}$ from the value of 1 used here. 

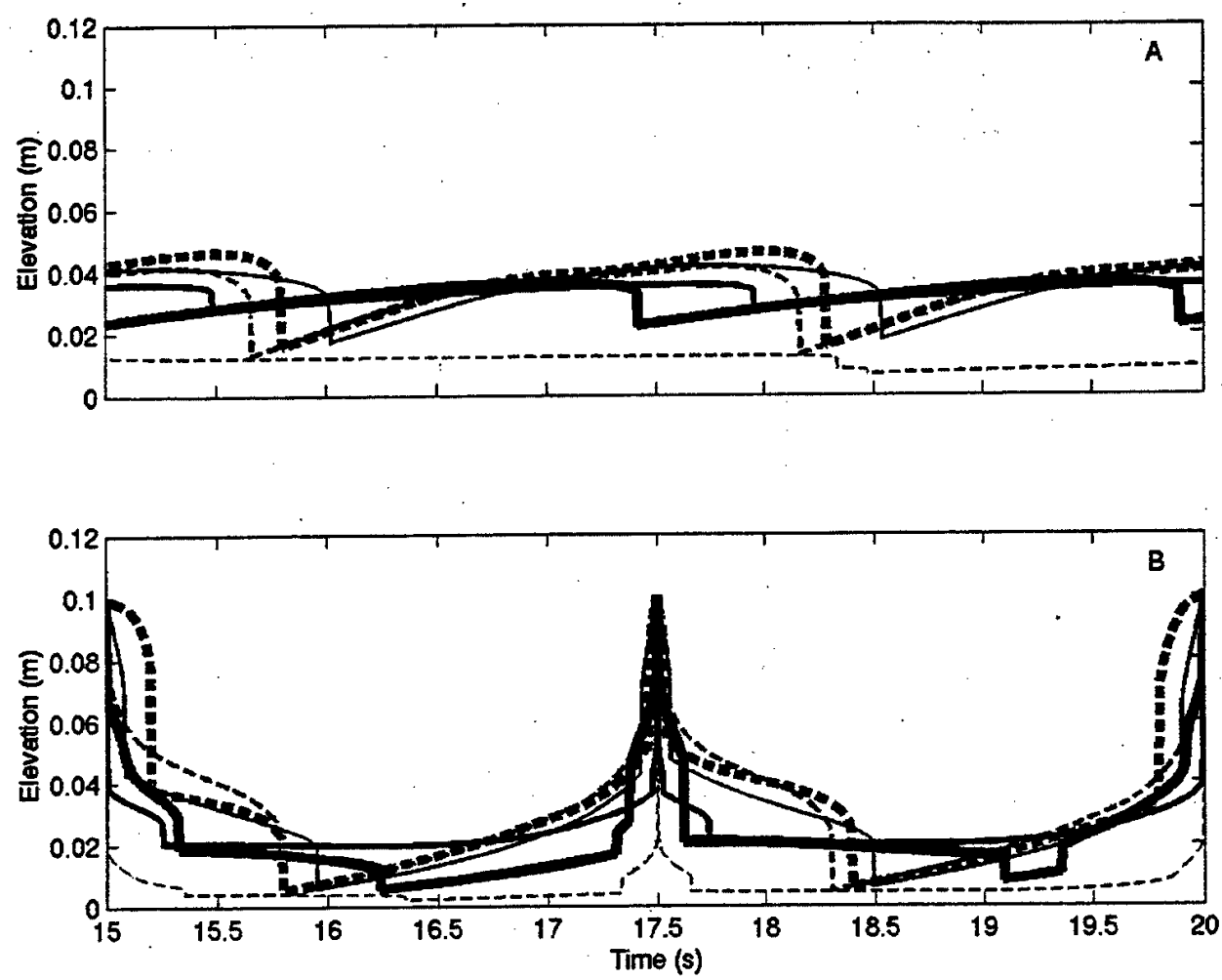

Fig. 4 - CASE1 boundary layer estimations for BL2 (A) and BL1(B): laminar (...............); linear (..............); parabolic (.............); $k(\longrightarrow) ; k-\varepsilon(\longrightarrow) ; k-\omega(\square)$.

Bed shear stress estimates for CASE1 show variation over the period and between the different model formulations (Fig. 5). For instance, all stress estimates have the same shape, but vary in magnitude from a maximum of nearly $1 \mathrm{~kg} \mathrm{~m}^{-1} \mathrm{~s}^{-2}$ for the laminar simulation (Fig. 5; thin dashed curve) to just over $4.0 \mathrm{~kg}$ $\mathrm{m}^{-1} \mathrm{~s}^{-2}$ for the linear and eddy viscosity model simulations (Fig. 5; bold dashed curves). These simulations also show the phase lead of the bed shear stress with respect to the free-stream velocity. This is apparent because the free-stream velocity maxima occur at 16.25 and $18.75 \mathrm{~s}$ while the two peaks in the bed shear stress occur at 16.0 and $18.5 \mathrm{~s}$ for the linear and parabolic simulations and 15.625 and 18.125 for the laminar simulation. This represents a phase lead of $0.25 \mathrm{~s}\left(18^{\circ}\right)$ for the linear and parabolic simulations and $0.625 \mathrm{~s}\left(45^{\circ}\right)$ for the laminar simulation. The $45^{\circ}$ lead for the laminar simulation exactly matches the phase lead predicted by boundary layer theory (Eq. (3)) for sinusoidal wave forcing (Stokes 1845). 


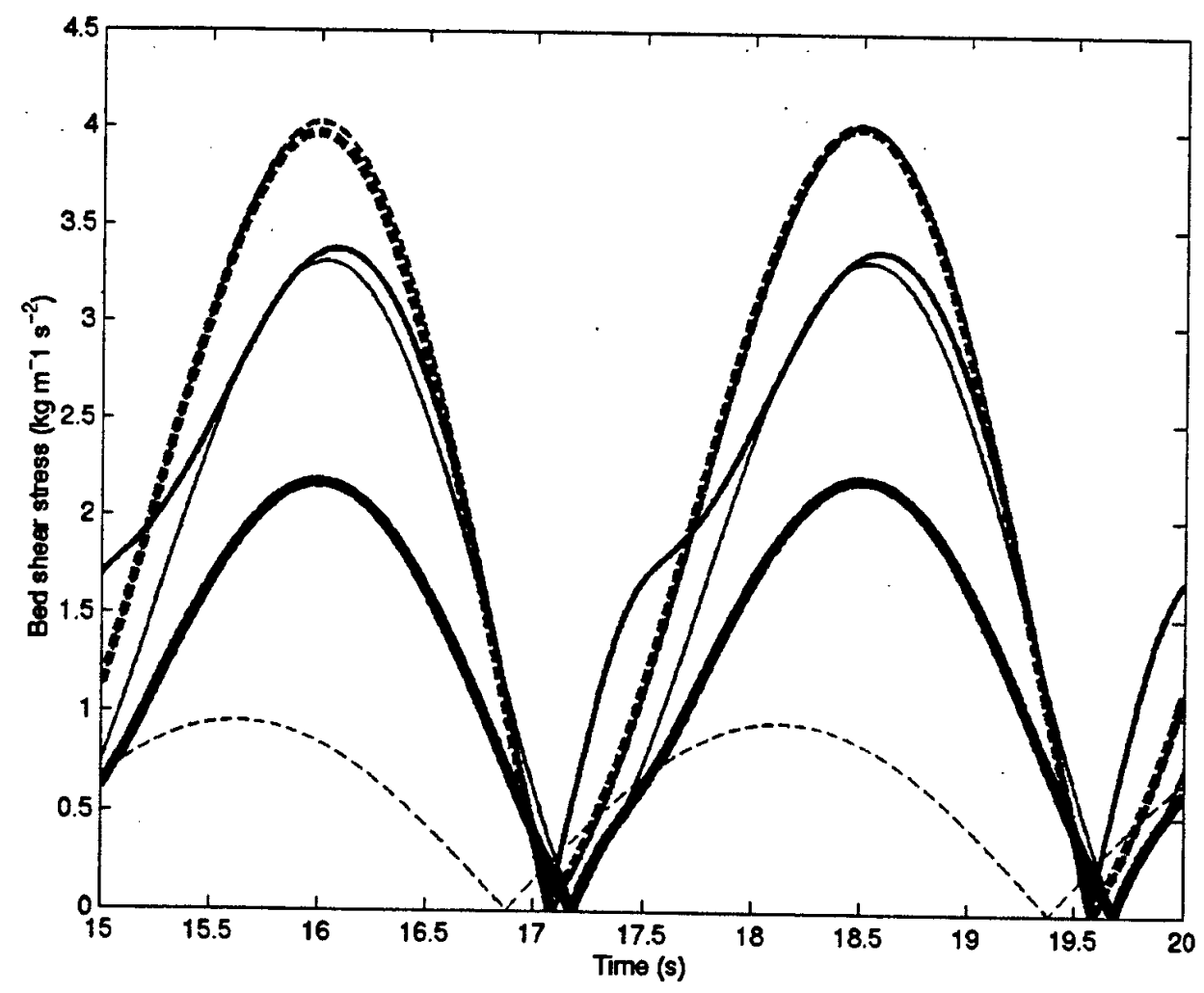

Fig. 5 - CASE1 bed shear stress estimate, laminar ( ); linear (.......); parabolic $(\mathbf{\square} \bullet \mathbf{\bullet} \mathbf{n})$; $k(\longrightarrow) ; k-\varepsilon(-)$

Bed shear stress estimates for the turbulent closure schemes (Fig. 5; solid curves) have the same shape as the eddy viscosity estimates but, interestingly, are generally smaller in magnitude. The phase lead of the bed shear stress of $15.8^{\circ}, 12.2^{\circ}$, and $18^{\circ}$ for the $k, k-\varepsilon$, and $k-\omega$ simulations, respectively, is similar to that of the linear and parabolic simulations and comparable to those given by Fredsce and Deigaard (1992, pg. 32) for amplitude Reynolds numbers ( $R E=\frac{U_{m} a}{v}, a=\frac{2 \pi U_{m}}{T}$ ) used here. The inflection in the $k-\varepsilon$ model bed shear-stress estimate is likely attributed to the flow reversal.

\section{MODEL-DATA COMPARISON}

Jensen et al. (1989) collected depth-dependent flow velocity measurements in a U-shaped oscillatory water tunnel for smooth and rough bed cases using a laser Doppler anemometer (LDA). The tunnel was $10 \mathrm{~m}$ long, $0.29 \mathrm{~m}$ wide, and $0.3 \mathrm{~m}$ deep $(0.28 \mathrm{~m}$ in smooth wall cases). The models will be tested against the Jensen et al. (1989) Test10 and Test13. Test13 was forced with a sinusoid of $9.72 \mathrm{~s}$, a maximum velocity of $2 \mathrm{~m} \mathrm{~s}^{-1}$ and a roughness of $K_{N}=0.84 \mathrm{~mm}$. Test 10 was forced with the same conditions except the bed was made smooth by the installation of PVC plates. Because the models require a $z_{0}\left(=K_{N} / 30\right)$ value, a smooth bed was approximated by using $K_{N}=1 \times 10^{-4} \mathrm{~mm}$ (four orders of magnitude smaller than the rough bed case and one order of magnitude smaller than the largest clay particle size on the Wentworth scale). Similar to the generic cases, 2000 time steps per wave period were used, and the number of grid points was 300 . 
Figures 6 and 7 show the velocity comparison for every 15 degrees of phase for the first half of the wave for the simulations (solid lines) and the Jensen et al. (1989) Test13 (dots). Each successive profile is offset by $2 \mathrm{~m} \mathrm{~s}^{-1}$ (denoted by the zeroes in the plot). The panels in Fig. 6 are A) laminar, B) linear, and C) parabolic. Similarly, the panels in Fig. 7 are A) $k, B$ ) $k-\varepsilon$, and C) $k-\omega$. The laminar simulation has difficulty matching the observed profile shape at all phases of wave motion:
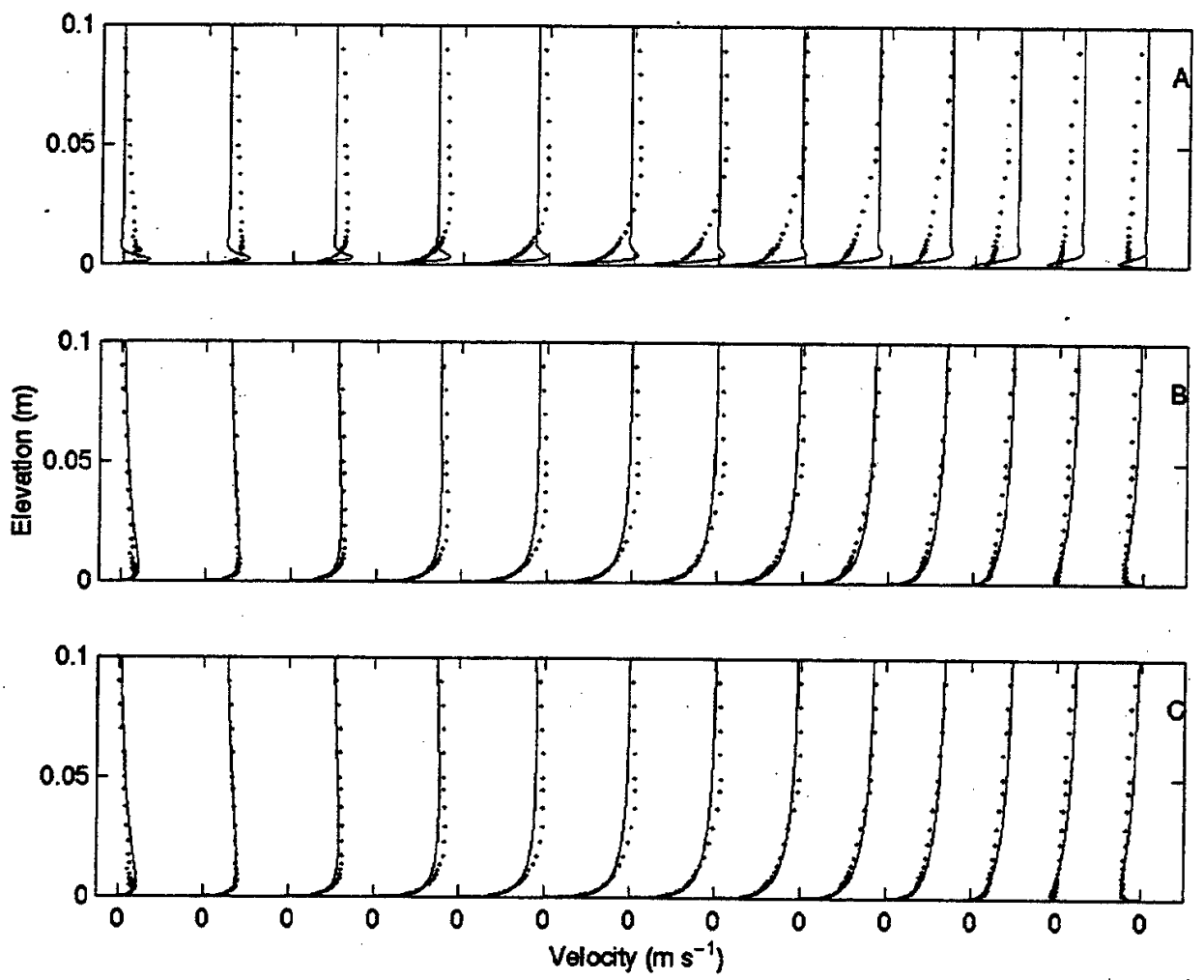

Fig. 6 - Rough wall (Test13), experimental data (.), model (solid curves). A) laminar, B) linear, and C) parabolic eddy viscosity. 

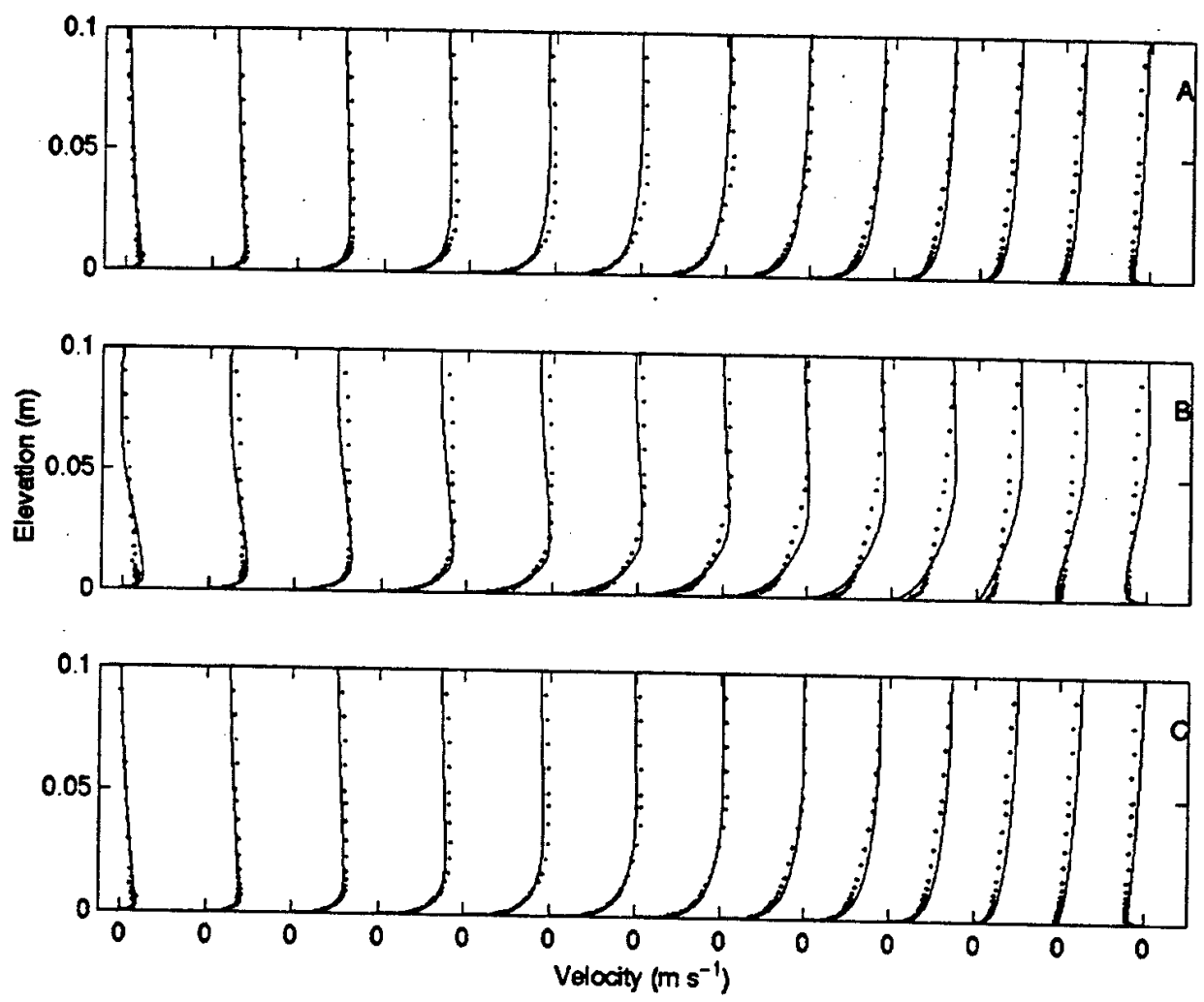

Fig. 7 - Rough wall (Test13), experimental data (.), and model (solid curves): A) $k$, B) $k-\varepsilon$, and C) $k-\omega$ turbulence models.

The $k-\varepsilon$ simulation generally has the correct velocity profile shape and magnitude in the upper portion of the domain. Near the bed, however, the $k-\varepsilon$ simulation only matches the shape of the observations, underpredicting the velocity magnitude at nearly all phases of the wave. The rest of the simulations are very similar and qualitatively match the data (quantitative comparisons are given below).

Figures 8 and 9 show the velocity comparisons to the data of Jensen et al. (1989) Test10 (layout is the same as in Figs. 6 and 7). The results are similar to the rough bed test in that the laminar simulation is the worst performer and the $k-\varepsilon$ simulation underpredicts the near-bed velocity at nearly all phases of wave motion. Figures 6 through 9 show that the simulations are qualitatively similar regardless of whether or not they are applied to rough or smooth beds. 

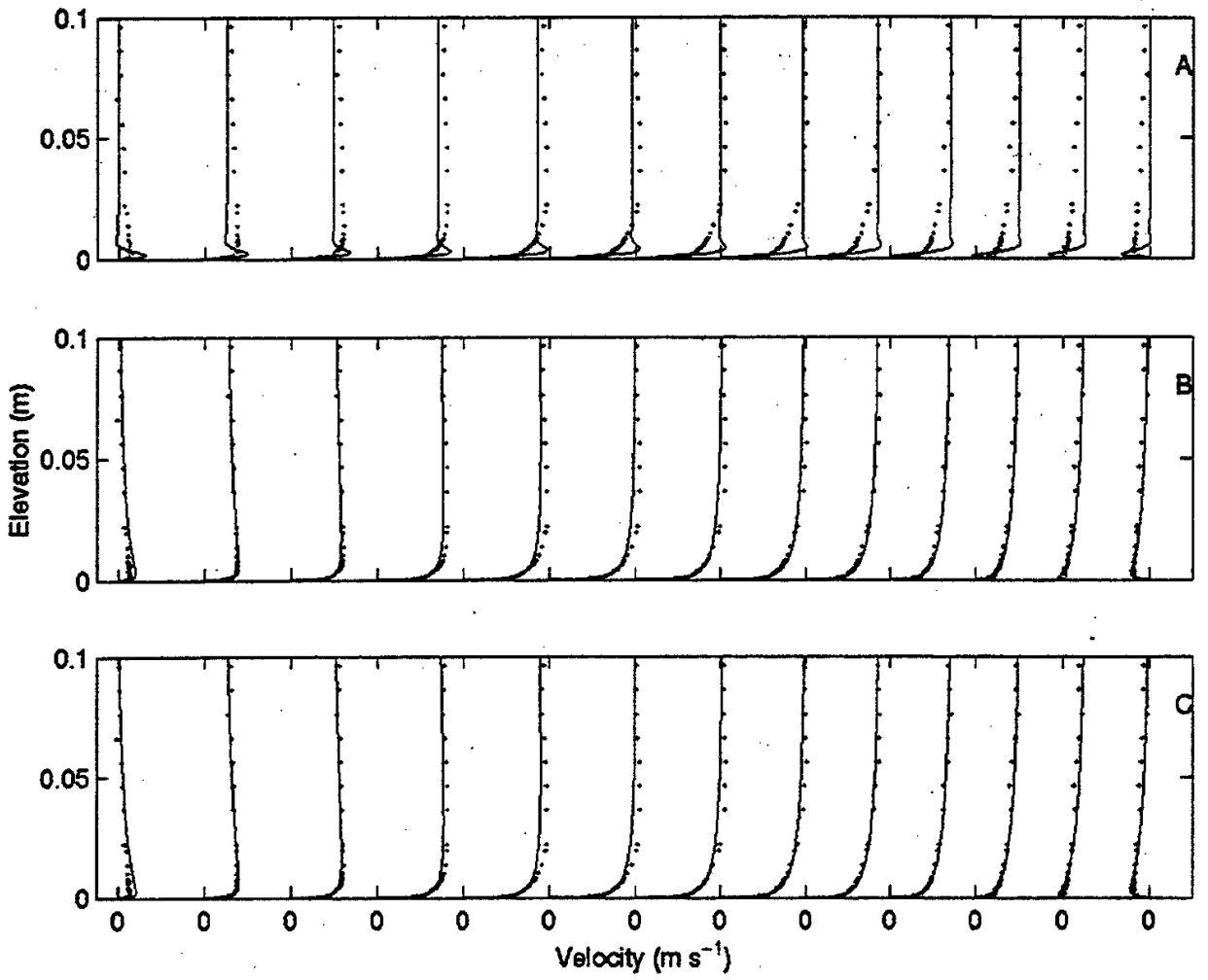

Fig. 8 - Smooth wall (Test10), experimental data (.), and model (solid curves): A) laminar, B) linear, and C) parabolic eddy viscosity.
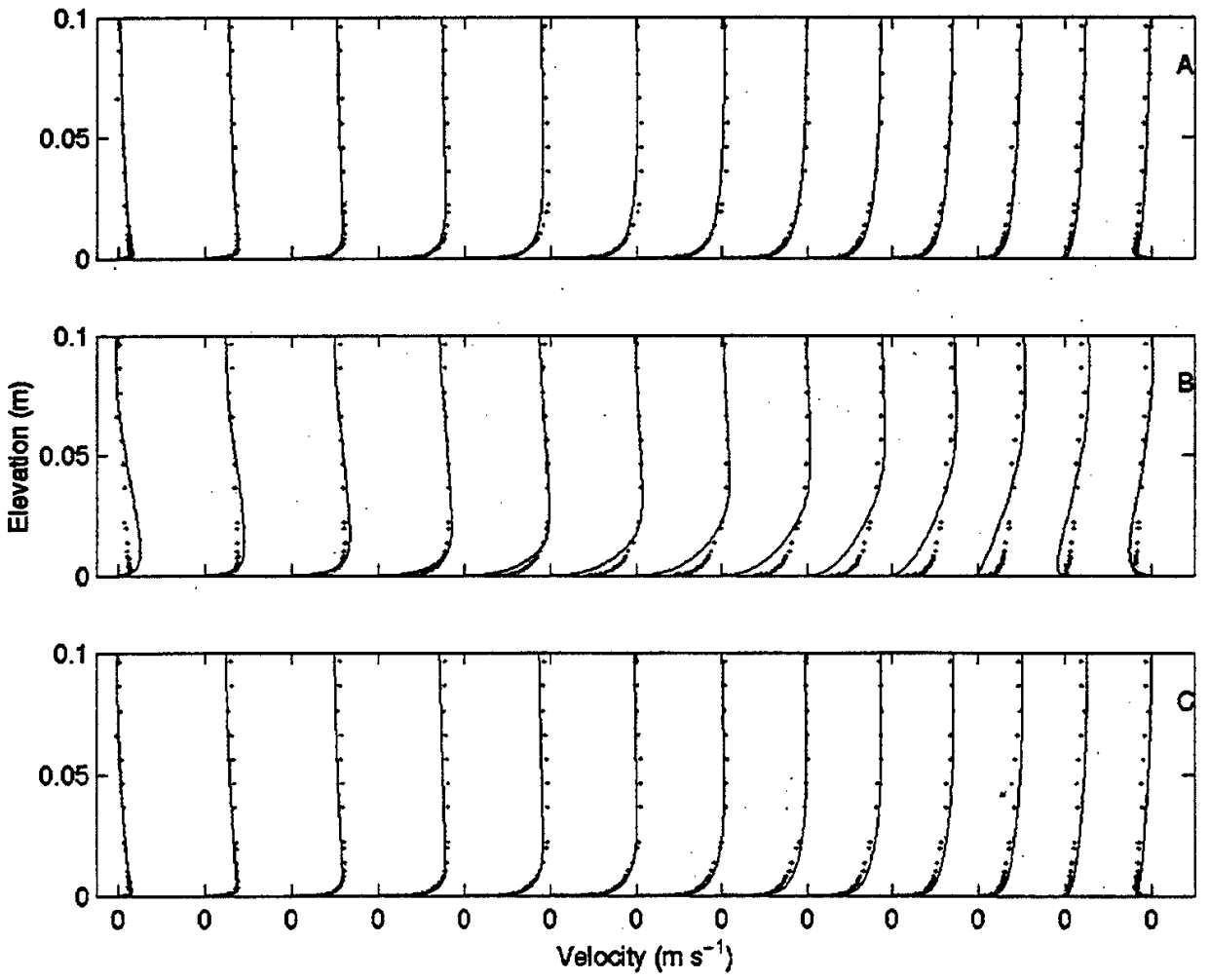

Fig. 9- Smooth wall (Test10), experimental data (.), and model (solid curves): A) $k, \mathrm{~B}) k-\varepsilon$, and C) $k-\omega$ turbulence models. 
A quantitative measure of the predictive capability of the models can be determined from the normalized time dependent error

$$
E_{p}=\sum_{z} \frac{\left(u_{\text {data }}-u_{\text {model }}\right)^{2}}{\sigma_{\text {data }}^{2}(t)}
$$

where $\sigma_{d a c a}^{2}(t)$ is the time-dependent vertical variance in data. $E_{p}$ values were calculated using the model grid points closest to the measurement locations. This distance was typically on the order of $1 \times 10^{-5} \mathrm{~m}$ or less except in the upper few centimeters of the domain where the maximum distance was $1.6 \times 10^{-3} \mathrm{~m}$. The smooth- and rough-bed comparisons show that the linear model was most accurate (although the parabolic, $k$, and $k$ - $\omega$ models had similar errors) while the laminar model was least accurate (see Fig. 10; laminar errors extend beyond the axis range shown). The $k-\varepsilon$ model had normalized errors that were up to six times as large as those of the $k$ and $k-\omega$ model. The largest errors are observed between $0^{\circ}$ and $20^{\circ}$ and between $160^{\circ}$ and $180^{\circ}$ near flow reversals. Away from flow reversals (adverse pressure gradients), all of the models except the laminar and $k-\varepsilon$ perform nearly equally for both the rough bed (Fig. 10A) and smooth bed (Fig. 10B) tests. In general, the best performers for both tests were the linear and parabolic eddy viscosity simulations and the $k-\omega$ model. Note the difference in scales on Figs. 10A and 10B, implying the models as a whole perform more poorly (by up to a factor of 3 ) for the smooth-bed simulation.
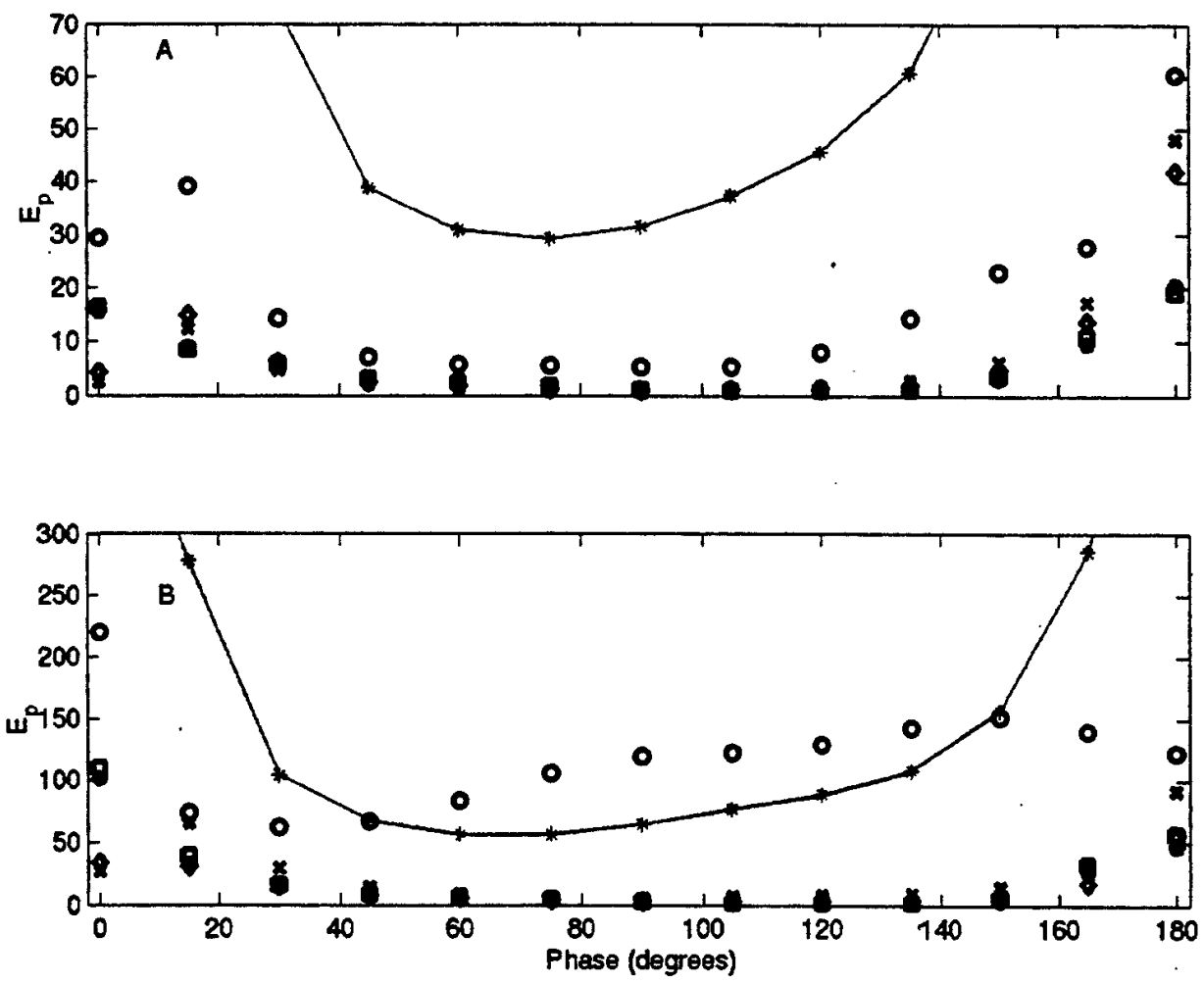

Fig. 10 - Normalized errors for rough-wall Test13 (A) and smooth-wall Test10 (B): laminar (gray *-); linear ( ${ }^{*}$ ); parabolic (square); $k$ (diamond); $k-\varepsilon(0)$; and $k-\omega(\mathrm{x})$. 


\section{DISCUSSION AND CONCLUSIONS}

The performance of seven boundary layer models has been investigated for two generic cases (sine wave and sawtooth wave) and data collected in a laboratory U-shaped oscillatory water tunnel. The eddy viscosity boundary layer models, where the vertical profile of the turbulent eddy viscosity is assumed to have a specific shape were similar in their predictive capability. Their similarity stems from the fact that within the boundary layer, the linear, linear-exponential, and parabolic forms for the eddy viscosity are also similar. The shapes of the eddy viscosity profile for these varies outside the boundary layer, but because there is little shear in the water column at these elevations, the differences have little effect on the predicted velocity profiles. The effect of a superimposed current is not included in the eddy viscosity models used here, but can be included through a modified eddy viscosity by either including the current effect in the bed shear stress estimate (e.g., Grant and Madsen 1979) or in a region-dependent (i.e., in the boundary layer or above) eddy viscosity model (e.g., Fredsøe and Deigaard 1992).

The accuracy for the turbulence closure schemes cannot be determined from the generic cases; rather, the attempt was to see how these models compared to the simplified, less numerically intensive eddy viscosity models. Based on these generic cases, it does not appear that the turbulence closure models yield velocity profiles that differ much from the eddy viscosity models. There was discrepancy, however, between the predicted bed shear stress between the two model types, with the eddy viscosity models predicting larger values than the turbulence closure schemes. These differences stem from the fact that the eddy viscosity models have a predefined shape for the eddy viscosity whereas the turbulence closure models do not.

The comparison to laboratory data showed how accurately the eddy viscosity and turbulence closure models, with the exception of the $k-\varepsilon$ model, predicted the observations. For instance, the $k$ and $k-\omega$ models performed well at all phases of the wave. The eddy viscosity models also compared well to the laboratory data for smooth and rough beds except, at times, in regions of flow reversal. The consistently larger errors associated with the $k-\varepsilon$ model result from the inability of the model to compensate at later phases for the overprediction during flow reversal. It has been suggested and shown previously (Rodi and Scheuerer 1986 and Wilcox 1988) that the $k-\varepsilon$ model can perform poorly during flow reversal (in regions with an adverse pressure gradient) as was observed. A typical workaround that was shown here, use of the $k$ - $\omega$ model, has better predictive capability during these times (Wilcox 1988).

The WBBL models presented here are a means by which vertical velocity profiles and bed shear stresses can be generated for a variety of analytic wave forms and free-stream velocity time series. The purpose of the models is to provide a simple means to estimate these parameters under a variety of conditions with the hopes of improving the knowledge of the fluid flow within the boundary layer and potentially enhancing sediment transport predictive capability. Based on this analysis, it does not appear that the extra computation time for the turbulence closure schemes (six times slower than the eddy viscosity models for the number of time steps used here) affords improvement in predictive capability. In conditions with much higher Reynolds numbers (i.e., cases with much larger velocities), stronger adverse pressure gradients, and/or 2D or 3D simulations, the use of the turbulence closure models may be more appropriate.

\section{ACKNOWLEDGMENTS}

This study was supported by Office of Naval Research (ONR) through base funding to the Naval Research Laboratory (PE\#61153N) (for J.A. Puleo) and ONR Coastal Geosciences Program and the National Science Foundation (for O. Mouraenko). B. Mutlu Sumer provided the LDA data. 


\section{REFERENCES}

Beach, R.A., and R.W. Sternberg, 1988. "Suspended Sediment Transport in the Surf Zone: Response to Cross-Shore Infragravity Motion," Marine Geology 80, 61-79.

Fredsøe, J., and R. Deigaard, 1992. Mechanics of Coastal Sediment Transport (World Scientific Publishing Co., River Edge, NJ).

Gelfenbaum, G., and J.D. Smith, 1986. "Experimental Evaluation of a Generalized Suspended Sediment Transport Theory," in Shelf Sands and Sandstones, pp. 133-144, American Society of Petroleum Geologists.

Grant, W.D., and O.S. Madsen, 1979. "Combined Wave and Current Interaction with a Rough Bottom," J.Geophys. Res. 84 (C4), 1797-1808.

Hwang, R.R., and S. Jaw, 1998. "Second-order Closure Turbulence Models: Their Achievements and Limitations, Proceedings of the National Science Council 22 (6), 703-722.

Jensen, B.L., B.M. Sumer, and J. Fredsoe, 1989. "Turbulent Oscillatory Boundary Layers at High Reynolds Numbers," J. Fluid Mechanics 206, 265-297.

Jonsson, I.G., 1966. "A New Approach to Oscillatory Rough Turbulent Boundary Layers," in Proceedings 10th Coastal Engineering Conference, pp. 127-148.

Justesen, P., 1988. "Prediction of Turbulent Oscillatory Flow Over Rough Beds," Coastal Engineering 12, 257-284.

Kajiura, K., 1968. "A Model of Bottom Boundary Layer in Water Waves," Bulletin Earthquake Res. Inst. Univ. Tokyo 46, 75-123.

Moneris, S.S., and D.N. Slinn, (submitted). "Numerical Simulation of the Wave Bottom Boundary Layer Over a Smooth Surface. Part 1: Three-Dimensional Simulations," J. Geophys. Res.

Pope, S.B., 2000. Turbulent Flows (University Press, Cambridge, UK).

Rodi, W., 1980. Turbulence Models and Their Application in Hydraulics (IAHR Delft, The Netherlands).

Rodi, W., and G. Scheuerer, 1986. "Scrutinizing the k- $\varepsilon$ Turbulence Model Under Adverse Pressure Gradients," ASME Journal of Fluid Engineering 108, 174-179.

Spalart, P., 1988. "Direct Numerical Simulation of a Turbulent Boundary Layer up to $\mathrm{R}_{\theta}=1410$," J. Fluid Mechanics 187, 61-98.

Stokes, G.G., 1845. "On the Theories of the Internal Friction of Fluids in Motion," Transactions of the Cambridge Philosophical Society 8, 287-305.

Wilcox, D.C., 1988. "Reassessment of the Scale-determining Equation for Advanced Turbulence Models," ALAA Journal 31, 1414-1421.

Wilcox, D.C., 2000. Turbulence Modeling for CFD (DCW Industries, Inc., La Canada, CA). 


\section{Appendix}

\section{MATLAB CODES}

The Matlab (written using Matlab 6.0) codes shown in Table A1 are included on the accompanying disk for convenience to the reader. The codes are heavily commented to explain the procedure throughout. The included setvars.m file will perform the simulation referred to as CASE1 for linear eddy viscosity. Every variable is described after it is defined and the recommended values are used. As an example, at the Matlab prompt, type: setvars('casel'). Then type evmodel('casel'). To see a quick plot of the velocity, load casel.mat and type plotmodel.

Table Al - Matlab Codes Used for Modeling the WBBL

\begin{tabular}{|l|l|}
\hline setvars.m & $\begin{array}{l}\text { Sets up the variables and constants for a model run. Used for all } 4 \text { model.m } \\
\text { files }\end{array}$ \\
\hline evmodel.m & $\begin{array}{l}\text { The WBBL model for the laminar, linear, linear-exponential, and parabolic } \\
\text { eddy viscosity model cases }\end{array}$ \\
\hline kmodel.m & The WBBL model using the one-equation turbulence model \\
\hline kemodel.m & The WBBL model using the two-equation $k-\varepsilon$ turbulence model \\
\hline kwmodel.m & The WBBL model using the two-equation $k-\omega$ turbulence model \\
\hline plotmodel.m & Plots the velocity profiles for the last wave in the simulation at 45 -deg intervals. \\
\hline
\end{tabular}

